\title{
Indicadores de inovação: um exame das atividades inovativas na indústria internacional de alimentos processados*
}

\author{
Vinicius C. B. Fornari** \\ Rogério Gomes*** \\ André Luiz Correa****
}

Recebido: 18/10/2013 Versão Revisada (entregue): 09/07/2014 Aprovado: 14/08/2014

\section{RESUMO}

Este artigo procura combinar métodos tradicionais de medição de intensidade tecnológica com outros indicadores alternativos para examinar a dispersão das atividades inovativas na indústria e em diferentes países. A hipótese que sustenta o estudo reside no fato de que nas indústrias tradicionais, como a de alimentos processados (IAP), os métodos tradicionais são insuficientes para detectar o processo de inovação em sua totalidade. Como método, foram analisados os dados de patentes extraídos das 25 maiores empresas de alimentos processados do mundo e propostos diferentes indicadores elaborados a partir da Pesquisa de Inovação Tecnológica - Pintec (IBGE, 2010) - empresas brasileiras - e da Community Innovation Survey - CIS (EUROSTAT, 2009) - países da União Europeia. Os resultados permitiram estabelecer relações em três dimensões: (i) a complexidade do esforço inovativo da IAP; (ii) os esforços para a inovação nos diferentes países são distintos; e (iii) existe heterogeneidade no desempenho dos países.

\footnotetext{
* Este trabalho foi desenvolvido no âmbito do Grupo de Estudos em Economia Industrial (GEEIN/UNESP). Os autores agradecem ao apoio da CAPES e as relevantes sugestôes dos pareceristas anônimos.

** Universidade Estadual de Campinas (Unicamp), Campinas (SP), Brasil. E-mail: viniciuscbfornari@yahoo.com.br

*** Universidade Estadual Paulista (Unesp), Araraquara (SP), Brasil. E-mail: rgomes@fclar.unesp.br

**** Universidade Estadual Paulista (Unesp), Araraquara (SP), Brasil. E-mail: andrelc@fclar.unesp.br
} 
Palavras-CHAVE | Difusão Tecnológica; Atividades Inovativas; Indústrias de Alimentos Processados

Códigos JEL | O33; O32; O31

\section{Innovation indicators: a survey of innovative activities in the international food processed industry}

\section{ABSTRACT}

This paper seeks to combine traditional methods of measuring intensity with other alternative indicators to examine the dispersion of innovation activities in different industries and countries. The hypothesis that underlies the study lies in the fact that in the Food Processed Industry (IAP) the traditional methods are insufficient to detect the core of the innovation process. As method, we analyzed patent data extracted from the twenty-five largest food processed companies in the world and suggested different indicators developed from the Pesquisa de Inovação Tecnológica (PINTEC, 2010) - for Brazilian companies - and the Community Innovation Survey (CIS, 2009) - for European Union companies. The results allowed us to establish relationships in three dimensions: (i) the complexity of the innovative effort of the IAP; (ii) the efforts to innovation in different countries are distinct and; (iii) there is heterogeneity in country performance.

KeYwords | Technological Diffusion; Innovative Activity; Food Processed Industry

JEL-CODES | O33; O32; O31 


\section{Introdução}

As invenções e inovações somente adquirem importância econômica em função de sua introdução no mercado e ampla difusão (ROSENBERG, 1982, p. 96). Nesse processo, as indústrias classificadas como baixa e média-baixa (BeMB) tecnologias (OCDE, 2003) têm papel fundamental como agentes difusores das inovações desenvolvidas por outras indústrias, em especial aquelas consideradas de média-alta e alta (MAeA) tecnologias. Nessa perspectiva, quanto mais densas as interações entre firmas com dinâmicas tecnológicas distintas, maior é estímulo ao crescimento e desenvolvimento econômico.

Os estudos sobre a inovação em indústrias de BeMB tecnologias estão concentrados em dois fenômenos complementares: o processo de adaptação e o de difusão tecnológica (ROBERTSON et al., 2009). Este último contempla as inovações incrementais, o desenvolvimento de habilidades pelos usuários, as complementaridades entre as diferentes técnicas de produção, o aperfeiçoamento de novas e velhas tecnologias e as mudanças no contexto institucional (FURTADO, 2006). Por outro lado, a adaptação inclui o desenvolvimento de capacitações pelas empresas para absorver e incorporar tecnologias.

Assim, o processo inovativo nas indústrias de BeMB tecnologias é caracterizado, principalmente, por inovações incrementais e "arquitetônicas", ambas relacionadas ao desenvolvimento de tecnologias existentes. As primeiras podem ser definidas como aprimoramentos e avanços tecnológicos em um produto e/ou processo. Já as inovações "arquitetônicas" são entendidas como combinaçôes de conhecimentos e/ou reconfiguração de tecnologias já existentes, visando obter novos produtos ou processos em resposta ao surgimento de novas oportunidades e/ou problemas técnicos-econômicos (HIRSCH-KREINSEN, 2008; HENDERSON; CLARK, 1990).

Entre as indústrias de BeMB tecnologias, este artigo destaca a indústria de alimentos (classe C10 da CNAE 2.0), mais apropriadamente denominada de indústria de alimentos processados (IAP), em função da importância da manufatura nessa atividade (SMITH, 2000). Além de participação expressiva na indústria de transformação em termos de emprego e produto, ${ }^{1}$ a IAP incorpora uma cadeia produtiva composta por inúmeros agentes em diferentes atividades econômicas. O processo de inovação é também bastante complexo, congregando desde esforços internos até aquisição de

1 Em 2008, em relação ao total da indústria de transformação, a IAP da Alemanha, França, Itália e Brasil responderam por 6,9\%, $12,5 \%, 10 \%$ e $16,2 \%$ das vendas e $8,3 \%, 11 \%, 7,4 \%$ do emprego, respectivamente (CIS, 2009 e PINTEC, 2010). 
tecnologias externas (exigindo da firma capacidade de assimilação), que possibilitam a diferenciação de produtos e, por vezes, o ingresso em novos mercados com bases tecnológicas novas ou complementares. Não obstante, como será visto, esse processo difere de acordo com as características das empresas e dos países onde atuam. Nesta perspectiva, a hipótese que sustenta o estudo reside no fato de que na IAP os métodos tradicionais de medição tecnológica (patentes ou gastos com P\&D) são insuficientes para detectar a essência do processo inovativo. Por isso, o estudo procura combinar os métodos tradicionais com a avaliação de indicadores alternativos elaborados a partir de dados da Pesquisa de Inovação Tecnológica - Pintec (IBGE, 2010) e da Community Innovation Survey - CIS (EUROSTAT, 2009), para empresas brasileiras e dos países da União Europeia, respectivamente.

Com esse intuito, a seção 2 destaca os elementos relevantes ao desenvolvimento tecnológico da IAP. Nas seções seguintes, a partir da combinação de dados de patentes das maiores empresas da IAP (seção 3) e de indicadores de inovação e atividades inovativas (seção 4), são discutidas as principais características da inovação tecnológica dessa indústria em diferentes países. Por fim, nas conclusões, são avaliados alguns dos resultados.

\section{Atividades tecnológicas na indústria de alimentos processados}

A partir da distinção entre pesquisas (P) e desenvolvimento (D), Christensen (1995) separa os ativos envolvidos no desenvolvimento tecnológico em quatro grupos: pesquisa científica, que se relaciona ao estoque e desenvolvimento de conhecimento novo - tem origem no desenvolvimento das ciências básicas ou na ligação entre ciência básica e aplicada; inovação em processo, compreendendo as tecnologias de produção (equipamentos, integração de sistema e organização do trabalho); inovação de produto, referente a recursos e capacidades necessários às atividades ligadas ao aperfeiçoamento e/ou criação de novos produtos; e design estético, que engloba estética do produto, embalagens, marcas, entre outros aspectos relacionados à promoção e comercialização do produto - importantíssimos por se tratar da interação direta entre os produtores e clientes. O desenvolvimento tecnológico é, muitas vezes, adequadamente descrito em termos de uma dessas categorias e, por vezes, caracterizado como uma forma "híbrida" que integra duas ou mais delas (CHRISTENSEN, 1995). Afora os resultados referentes ao primeiro ativo, os demais são considerados componentes do desenvolvimento, cujos resultados inovativos poucas vezes são captados integralmente pelas estatísticas de P\&D ou patentes. 
FIGURA 1

Modelo interativo e atividades inovativas na IAP

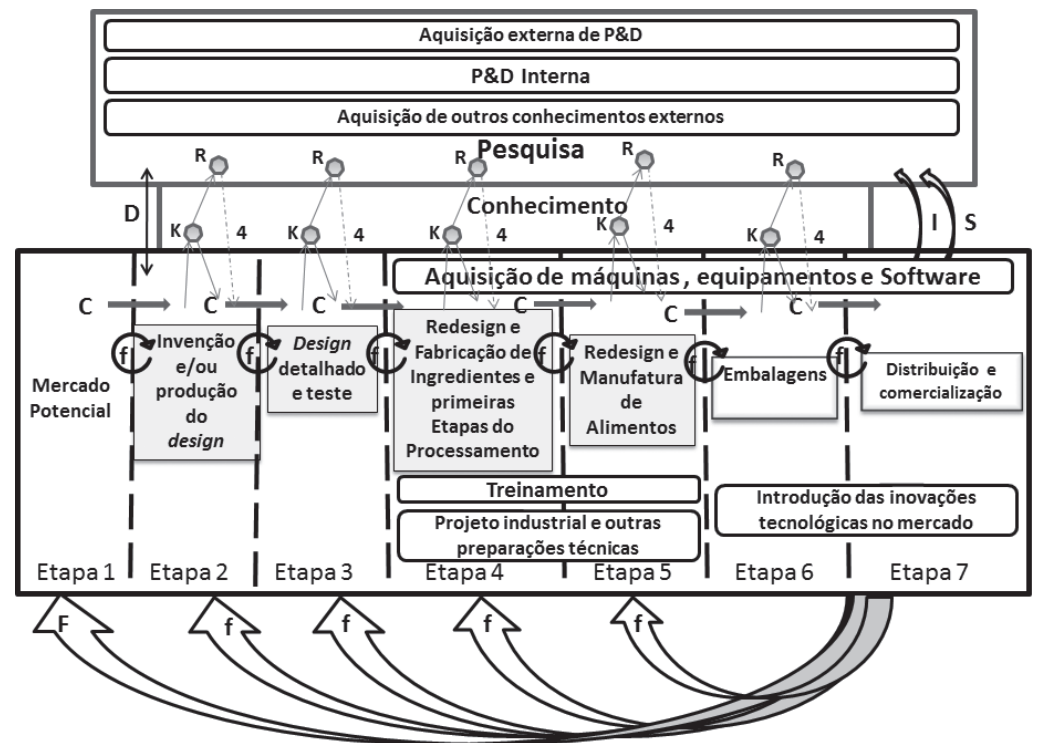

Legenda dos fluxos:

C: Caminho central de inovação.

F e f: Caminhos dos feedbacks, sendo F feedbacks particularmente importantes.

Fluxo K-R: Interação entre conhecimento e pesquisa. Se um problema é resolvido no nó K, a ligação 3 não é ativada. A ligação direta entre pesquisa e as demais atividades (ligação 4) não é simples e, por isso, tem linha pontilhada.

D: Ligação direta entre a pesquisa e os problemas de invenção e design.

I: Instrumentos, máquinas, ferramentas e procedimentos tecnológicos que dão suporte à pesquisa científica.

S: Suporte à pesquisa dado pela área de produto por meio de informaçôes diretas e pelo monitoramento externo (clientes, fornecedores, competidores, etc.). As informaçôes obtidas podem ser aplicadas em qualquer ponto ao longo da cadeia.

Os "balóes" procuram caracterizar as atividades inovativas da IAP em termos de etapas/tecnologias desenvolvidas nos departamentos.

Fonte: Kline e Rosenberg (1986), Smith (2000) e Acha e Tunzelmann (2005). Elaboração dos autores.

A Figura 1 procura ajustar o "modelo interativo de inovação" proposto por Kline e Rosenberg (1986) às especificidades da IAP e, ao mesmo tempo, contemplar, como proposto pelo Manual de Oslo (OECD, 2005) e incorporado nas publicações da Pintec e CIS, os sete tipos de atividades entendidas como tecnológicas (IBGE, 2008, p. 8): (1) atividades internas de P\&D (P\&D); (2) aquisição externa de P\&D (P\&De); (3) aquisição de outros conhecimentos externos (CE) - transferência de tecnologia pela compra de licença de direitos de exploração de patentes e uso de marcas; (4) aquisição de máquinas, equipamentos e software (AMES); (5) treina- 
mento (T); (6) introdução de inovações tecnológicas no mercado (IM); (7) projeto industrial e outras preparações técnicas para a produção e distribuição (PP).

A etapa 1 (mercado potencial) analisa as oportunidades tecnológicas e de mercado e define as diretrizes para o desenvolvimento de um produto novo ou aprimorado. Nas etapas 2 e 3, as inovações incrementais e de produto são mais constantes e, por isso, estão mais associadas à pesquisa do que as etapas subsequentes. Nelas, há o desenvolvimento do produto com preocupações nos elementos nutricionais, textura, cor e sabor.

Entre as três primeiras etapas, as atividades inovativas mais relevantes são de P\&D interna, aquisição de P\&D e outros conhecimentos externos, realizadas em cooperação com institutos de pesquisas e universidades, empresas da química, farmacêuticas, entre outras. Por exemplo, os alimentos funcionais ou nutracêuticos ${ }^{2}$ são produtos "dois em um", pois suprem as necessidades nutricionais básicas e também proporcionam benefícios à saúde, auxiliando no combate de doenças crônico-degenerativas ou corrigindo pequenas disfunções do organismo humano (doenças cardiovasculares, problemas intestinais, hipertensão, osteoporose, câncer, etc.). Outro caso são os alimentos "nutricosméticos", que, além de nutritivos, proporcionam benefícios à pele. Nestes casos, afora o contato próximo com os fornecedores para definir as especificações de insumos, a IAP precisa desenvolver capacitações e canais para assimilação desses conhecimentos e tecnologias. Ademais, nessas fases iniciam-se os estudos de engenharia de processo para a produção do novo produto.

A etapa 4 (redesigning e fabricação de ingredientes e primeiras etapas do processamento) é voltada para definir os sistemas de seleção e preparação das matérias-primas, incluindo atividades como lavagem, classificação, seleção, filtragem, etc. $\mathrm{Na}$ etapa 5 (redesigning e manufatura de alimentos), ocorre a adaptação do projeto do produto ao processamento/manufatura do alimento, que inclui uma vasta gama de funções de processamento, cozimento e pasteurização (SMITH, 2000). Nestas duas etapas, as atividades inovativas mais frequentes são as de aquisição de maquinas, equipamentos e software, treinamento e projetos industriais e outras preparaçóes técnicas, que estão relacionadas às inovações ou assimilação de tecnologia de processo e objetivam, principalmente, o aumento da produtividade e a diminuição dos custos operacionais. Estas atividades contemplam também interações (conhecimento externo complementar e aprendizado interno) da IAP com outras indústrias, como,

2 O termo nutracêutico vem de "nutri", nutriente, e "cêutico", de farmacêutico. 
por exemplo, com os fornecedores de máquinas e equipamentos (relação usuário-produtor).

A etapa 6 (embalagem), de acondicionamento e revestimento do produto, envolve técnicas de preservação e armazenamento (métodos de preservação químicos, biológicos, etc.), segurança alimentar (prevenção de todos os tipos de contaminação - seja dos produtos, seja do ambiente de produção - e seleção de equipamentos e materiais inofensivos) e ambiental (descarte, reciclagem, etc.) (SMITH, 2000). Nesta etapa, a atividade inovativa mais importante é a introdução das inovaçôes no mercado, tarefa relacionada a conservação, condições de transporte, marketing e publicidade do produto. O objetivo é evitar customização de produtos e, quando possível e vantajoso, fornecer versões adaptadas a nichos de mercados.

A última etapa (distribuição e comercialização) não é específica da indústria. No entanto, muitas empresas da IAP ingressaram nesta atividade por entendê-la como estratégica em virtude da perecibilidade de muitos dos bens vis-à-vis as longas distâncias entre os fornecedores de matérias-primas e produtores, entre produtores e clientes e consumidores (SMITH, 2000).

Por fim, assim como descrito por Kline e Rosenberg (1986), os mecanismos de feedback entre as etapas produtivas e tecnológicas permitem a interação entre os departamentos com intuito de alcançar os melhores métodos de produção, corrigir as possíveis falhas durante o processo produtivo e promover adaptações nos produtos, de forma a tornar sua produção viável. Considerado particularmente importante pelos autores, o feedback (F) auxilia no aprimoramento ou desenvolvimento de novos produtos em resposta aos sinais do mercado. Recordemos que na indústria de alimentos as preferências dos consumidores têm grande relevância na determinação dos produtos ofertados.

Outra característica do desenvolvimento tecnológico da IAP é a importância do conhecimento oriundo de outras instituições e áreas científicas. Esta indústria requer um conjunto amplo de conhecimentos com atuação de diferentes áreas da ciência (química, biologia, física, tecnologia de alimentos, biotecnologia, eletrônica, instrumentação, engenharia, etc.), aspectos discutidos na seção a seguir. Apesar dos níveis baixos de P\&D quando comparada a outras indústrias (OCDE, 2003), a IAP é uma das indústrias mais intensivas em conhecimento (SMITH, 2000, p. 28). 


\section{Patentes na indústria de alimentos processados}

A análise dos depósitos de patentes permite mensurar parte dos resultados das atividades de inovação das empresas que provêm da aquisição e do desenvolvimento da base de conhecimento codificado e ampliação das competências técnicas nos variados campos científicos. A partir de uma metodologia similar à utilizada por Patel e Pavitt (1997), esta seção procura complementar a anterior ao examinar as áreas científicas das patentes das grandes empresas da IAP para relacioná-las ao desenvolvimento tecnológico da indústria. Para tanto, extraíram-se todas as patentes da base ISI Web of Knowledge/Derwent Innovations Index - das 25 maiores empresas da IAP, ${ }^{3}$ no período de 1986 a 2010. Foram encontrados 15.928 registros de patentes, ${ }^{4} \mathrm{em}$ 252 campos distintos entre os 292 classificados a dois dígitos (83,6\% dos campos técnicos). Mesmo que uma invenção possa ser registrada em mais de um desses campos, a porcentagem é reveladora da multidisciplinaridade do conhecimento técnico necessária às grandes empresas da IAP.

Como recorte metodológico, definiu-se a frequência relativa (FR) como a proporção entre o número de registros de cada campo técnico e o total de patentes no período analisado (1986-2010):

$$
\text { FR(i) }(\%)=\frac{\text { Número de Registro do Campo(i) }}{\text { Número total de patentes }} \text {, }
$$

No intuito de delimitar o escopo do presente estudo, esta seção analisa apenas os campos técnicos da IAP ${ }^{5}$ com maior frequência relativa superior a $0,5 \%$. Desse procedimento restaram 38 ( $15 \%$ do total) campos técnicos que tiveram as patentes classificadas por um segundo critério: a Taxa de Crescimento Ponderada (TCP), uma variação do número de patentes registradas no campo técnico. Para evitar distorções entre o volume de registros de diferentes campos técnicos, a TCP foi calculada pelo somatório das taxas de crescimento quinquenais ( $\mathrm{t}=1986-90$; 1991-1995; 1996-

3 Nestlé (Suíça), Unilever (Holanda), Kraft Foods (EUA), Archer Daniels (EUA), Danone (França), Wilmar International (Cingapura), Bunge (Bermuda), General Mills (EUA), Kellogg (EUA), Assoc. British Foods (Inglaterra), HJ Heinz (EUA), Tyson Foods (EUA), ConAgra Foods (EUA), Sara Lee (EUA), JBS (Brasil), Campbell Soup, BRF-Brasil Foods (Brasil), Grupo Bimbo (México), Tingyi Holding (China), Orkla (Noruega), Golden Agri-Resources (Cingapura), Hershey (EUA), Ajinomoto (Japão), IOI Group (Malásia) e JM Smucker (EUA) - (FORBES, 2011).

4 Para efeitos de comparação, a Samsung, líder mundial do ramo eletrônico, registrou 11.692 patentes em 2010 (ISI, 2013).

5 Foram excluídos cinco campos técnicos não relacionados à IAP com frequência superior a 0,5\%: D25, D21 e D23 - cosméticos desinfetantes e detergentes; A96 - polímeros e plásticos, com aplicaçōes em produtos médicos, dentários, veterinários e cosméticos; e P24 - artigos de mão, de viagem e escovas. 
2000; 20001-05 e 2006-10) ponderadas pela participação do número de patentes do campo técnico (i) no total do respectivo quinquênio:

$$
\operatorname{TCP}(\mathrm{i})=\sum\left[\frac{\text { Número de Registros do Campo (i) no período (t) }}{\text { Número total de patentes, }} \times \text { Taxa de Crescimento do campo (i) entre (t) e (t-1) }\right]
$$

Os dois indicadores acima permitiram classificar os campos técnicos em quatro grupos segundo as respectivas medianas: centrais - destacam-se em termos de frequência e taxa de crescimento, ambas com percentuais acima das medianas; emergentes - baixa participação em termos de registros, mas elevado crescimento dos patenteamentos (inferior e superior à mediana, respectivamente); complementares inverso do anterior (superior e inferior à mediana, respectivamente); e marginais - as duas taxas são inferiores às medianas.

\section{GRÁFICO 1}

Distribuição dos campos técnicos

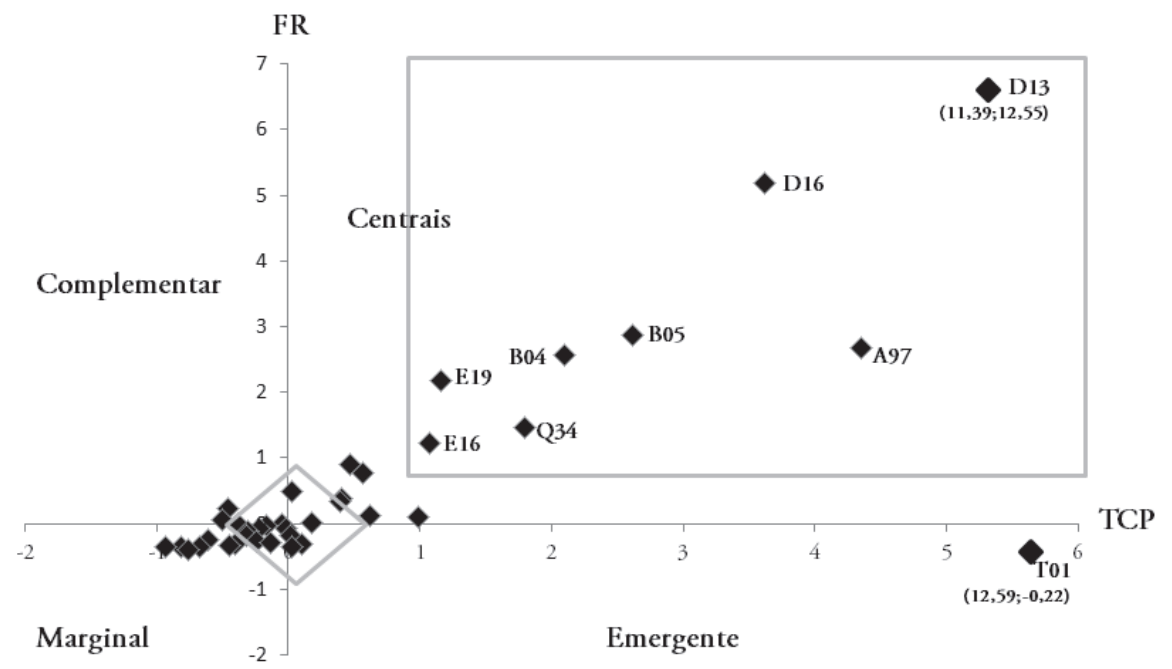

Fonte: ISI (2013). Elaboração dos autores.

Nota: Os valores da FR e da TCP foram normalizados pelas respectivas medianas. Os códigos são do campo técnico das patentes.

Os resultados obtidos por meio da metodologia descrita anteriormente são sintetizados no Gráfico 1. Foram encontrados 16 campos "centrais", dos quais são destacadas oito patentes circunscritas no retângulo do Gráfico 1. A primeira observação importante sobre este resultado pode ser formulada a partir do seguinte questionamento: como as firmas de alimentos, tradicionalmente interpretadas como 
de baixa intensidade de $\mathrm{P} \& \mathrm{D}$, podem sustentar a infraestrutura técnico-científica capaz de mantê-las "ativas" ao longo do tempo em tão diferentes e numerosos campos técnicos?

Além de parte expressiva dos depósitos de patentes ser realizada por empresas multinacionais, ${ }^{6}$ muitas inovaçôes ocorrem ao longo da cadeia de valor, especialmente pela interação técnica com fornecedores. As inovações em produtos, equipamentos e processos demandam expertises diversificadas, ou seja, em áreas não nucleares aos negócios - cerca de 50\% das inovações estão ligadas a agricultura, bioengenharia, química, farmacêutica, embalagens. Esse conhecimento variado permite que os grandes grupos usufruam de vantagens advindas de sinergias e economias de escopo em tecnologias nos diferentes ramos de atuação. No entanto, mesmo que as multinacionais difiram de outras empresas da IAP em termos de inovatividade, dotação e variedade tecnológica, a imitação é fácil. Assim, o aprendizado torna-se elemento central para o desenvolvimento da indústria (DOMINGUES, 2008, cap. 2).

Em destaque no Gráfico 1, os campos outros gêneros alimentícios e de tratamento (D13) - maior número de registros de patente em todos os quinquênios - e fermentação (D16) estão diretamente vinculados com as atividades da IAP. Já os campos associados com o desenvolvimento de alimentos nutracêuticos (B04 e B05), que segundo Raud (2008) são tecnologias da nova fronteira da indústria, tiveram aumento de registros principalmente nos anos 2000.

Apesar das frequências e taxas de crescimentos menores que os anteriores, outros campos técnicos destacados no Gráfico 1 (vide retângulo) são auxiliares no desenvolvimento tecnológico da IAP. A química orgânica (E16 e E19) é importante no desenvolvimento de produtos e insumos e para as duas classes mencionadas acima (D1 e B). Mesmo não sendo áreas específicas das tecnologias de alimentos, os polímeros e plásticos (A97) e os elementos de embalagem (Q34) são fundamentais na preservação e transporte dos produtos.

A quantidade de campos técnicos em torno das medianas (vide losango), que contemplam variados conhecimentos científicos necessários para a IAP, é outro particular do Gráfico 1. Parte expressiva dos campos complementares (Q31, B02, B03, E12 e E14), emergentes e marginais, que envolvem conhecimentos heterogêneos associados a diferentes etapas da cadeia de valor, está concentrada naquela região: engenharia (P34 e P73), elétrica (X27), embalagens (Q33), polímeros naturais (A11) e específicos da IAP - tratamento e processamento de carne de ave ou peixe (D12).

6 Segundo Alfranca, Rama e Von Tunzelmann (2005), estas empresas são responsáveis por $50 \%$ do total de patentes da IAP. 
Todas essas competências são importantes, pois, além de facilitarem a incorporação de tecnologias dos fornecedores, são essenciais para o desenvolvimento das inovações guiadas pelo usuário (VON HIPPEL, 2005).

Outro aspecto a destacar é a concentração de patentes em poucas empresas da amostra. Do total de registros, $87,7 \%$ pertencem a apenas quatro grupos: Unilever (43,1\%), Ajinomoto (32,6), Kraft Foods $(7,9 \%)$ e - a maior da amostra - Nestlé $(4,1 \%)$. Nesse sentido, as patentes são ativos complementares e uma política específica de cada uma das grandes empresas da indústria. Além do patenteamento, o segredo industrial, a gestão da marca, tradição e reputação, qualidade dos produtos e pequenas inovações cotidianas ao longo da cadeia de valor também são estratégias de mercado e tecnológicas na IAP (FILIPPAIOS; RAMA, 2011). Ademais, o baixo patenteamento e a concentração em poucas empresas podem decorrer também das estratégias de diferenciação por meio de lançamentos frequentes de produtos com pequenas modificações técnicas (sabor, textura, cor, etc.), dos esforços em inovações visando a adaptação do produto ao mercado local (FILIPPAIOS; RAMA, 2011; ALFRANCA et al., 2005) e da diversificação dos ramos de negócios.

O exame das patentes das quatro empresas acima constata que há diferenças nos resultados da produção de novos conhecimentos em cada uma delas. Apenas os campos diretamente ligados à IAP (D13 e D16) estão entre os "centrais", ou seja, apresentaram elevado volume de registros em todos os períodos. A Unilever (231) e Ajinomoto (219) possuem o maior número de patentes. A primeira companhia tem elevados percentuais de patentes em campos relacionados às estratégias de diversificação (PENROSE, 1959, cap. 7), que não fazem parte da IAP (D25, D21 e A96, todos desconsiderados no Gráfico 1 - vide nota 5). Já o caso da Ajinomoto é distinto, pois a empresa parece manter a produção de tecnologias concentrada nos campos considerados "centrais" (principalmente nas classes D1 e B) e poucos registros vinculados à diversificação (D21). Por fim, apesar de dispersas em campos técnicos variados, as patentes da Kraft Foods e da Nestlé concentram-se nos campos "centrais" (principalmente D1).

Em suma, o exame dos registros de patentes permite admitir que a incorporação de conhecimento de diferentes áreas científicas - para criar, aperfeiçoar e diferenciar produtos e diversificar os negócios - é absolutamente compatível com o modelo interativo da seção anterior. Todavia, como a análise de patentes é restrita a poucas e grandes empresas, esses registros não abarcam todos os esforços inovativos (MENDONÇA, 2009). Os indicadores da próxima seção procuram superar essa limitação. 


\section{Indicadores de inovação e de atividades inovativas na indústria de alimentos processados}

Os indicadores tecnológicos desta seção foram desenvolvidos com o objetivo de mensurar os esforços, avaliar os resultados da inovação na IAP e discutir as diferenças entre os países. Nessa perspectiva, o conjunto de indicadores deste item foi elaborado a partir do número de empresas ${ }^{7}$ e, para efeitos comparativos, aplicados a um conjunto de países (Brasil, Alemanha, Espanha, França, Itália, Holanda, EU-14 ${ }^{8}$ ). Esses indicadores foram divididos em classes com a intenção de expressar três aspectos distintos da inovação: resultados - avaliam os tipos de inovações; dimensão - procura intuir a abrangência das inovaçōes; e esforços - as "práticas" segundo os diferentes tipos de atividades. As classes ${ }^{9}$ e os indicadores propostos estão descritos no Quadro 1.

Os indicadores foram desenvolvidos a partir das informações obtidas na Pintec, (IBGE, 2010) e CIS (EUROSTAT, 2009). ${ }^{10}$ O Manual de Oslo (OECD, 2005, p. 23), guia para estas publicaçôes, considera apenas empresas comerciais e entende como inovações as alterações: nas potencialidades dos produtos - bens e serviços - que os tornem novos ou significativamente melhorados ou aperfeiçoados para empresa ("novo para a firma") ou mercado; nos processos e distribuição; nas práticas de negócio; na organização do local de trabalho ou nas relações externas das empresas; nos métodos de marketing (no design do produto e da embalagem, na promoção do produto e sua colocação); e em métodos de estabelecimento de preços de bens e de serviços.

Para o exame dos indicadores de resultados e dimensão da inovação (Quadro 1), a subseção a seguir utiliza estatística descritiva associada ao exame do coeficiente de variação $(\mathrm{CV}),{ }^{11}$ empregado para avaliar a heterogeneidade dessas taxas. $\mathrm{Na}$

7 Ao contrário do tradicional (uso de indicadores de intensidade), os indicadores aqui utilizados buscam expressar a difusão ("dispersão") das atividades tecnológicas na indústria. As hipóteses subjacentes é que a dispersão permite hierarquizar cada uma das atividades praticadas (relevância) e caracterizar o processo inovativo de cada país.

8 EU-14: agrega 14 outros países da União Europeia, além dos citados, com informaçôes completas disponíveis na CIS: Áustria, Bélgica, Bulgária, Croácia, Eslováquia, Finlândia, Hungria, Malta, Noruega, Polônia, Portugal, República Checa, Romênia e Suécia.

9 As três classes de indicadores propostos são consistentes com a aplicação do método de análise fatorial por componentes principais. Os resultados deste exercício não são exibidos aqui por razões de espaço, mas poderão ser disponibilizados mediante solicitação.

10 As referências a estas duas pesquisas são pelo ano de publicação, mas os indicadores (Quadro 1) de resultados e dimensão da inovaçăo referem-se aos dados de 2006 a 2008 e os de esforços ao ano de 2008.

$11 \mathrm{O}$ coeficiente de variação de Pearson $(\mathrm{CV})$, a razão entre o desvio padrão e a média, permite comparaçōes entre variáveis de naturezas distintas e fornece uma ideia da dispersão relativa dos dados. Considera-se que quanto menor o coeficiente, mais homogêneos serão os dados. Aqui adotamos taxas inferiores a $25 \%$ como critério para "homogêneo". 
avaliação dos indicadores de esforços, é feito um exercício de análise de agrupamento para hierarquizar essas atividades e, também, avaliar a similaridade dos países com respeito às atividades inovativas.

\section{QUADRO 1}

Síntese dos indicadores de inovação propostos

\begin{tabular}{|c|c|c|}
\hline Avaliação & Indicador & Definição \\
\hline \multirow{10}{*}{$\begin{array}{l}\text { Resultados } \\
\text { das inovaçóes }\end{array}$} & \multirow{10}{*}{$\begin{array}{l}\text { Taxa de inovação } \\
(\mathrm{TI})= \\
\text { Taxa de inovação } \\
\text { em processo } \\
\text { (TIPr) = } \\
\text { Taxa de inovação } \\
\text { em produto } \\
\text { (TIP) = } \\
\text { Taxa de inovação } \\
\text { organizacional } \\
\text { e/ou marketing } \\
\text { (TIOM) = }\end{array}$} & Número de empresas que inovaram (produto e/ou processo) \\
\hline & & Número total de empresas da amostra \\
\hline & & \\
\hline & & Número de empresas com inovação em processo \\
\hline & & Número total de empresas da amostra \\
\hline & & Número de empresas com inovação em produto \\
\hline & & Número total de empresas da amostra \\
\hline & & \\
\hline & & Empresas com inovaçôes organizacionais e/ou marketing \\
\hline & & Número total de empresas da amostra \\
\hline \multirow{4}{*}{$\begin{array}{l}\text { Dimensão da } \\
\text { inovação }\end{array}$} & \multirow{4}{*}{$\begin{array}{l}\text { Produto novo } \\
\text { para empresa } \\
(\mathrm{IPE})= \\
\text { Produto Novo } \\
\text { para Mercado } \\
(\mathrm{IPM})=\end{array}$} & Empresas que desenvolveram inovaçóes em produtos novos para a empresa \\
\hline & & Número de empresas que desenvolveram inovaçóes \\
\hline & & Empresas que desenvolveram inovações em produtos novos para o mercado \\
\hline & & Número de empresas que desenvolveram inovaçóes \\
\hline \multirow{2}{*}{$\begin{array}{l}\text { Esforços para } \\
\text { Inovação } \\
\text { (Tipos de } \\
\text { Atividades } \\
\text { Inovativas) }\end{array}$} & \multirow[b]{2}{*}{$\begin{array}{l}\text { Taxa de atividade } \\
\text { inovativa (i) }=\end{array}$} & Número de empresas que realizaram a atividade inovativa(i) \\
\hline & & $\begin{array}{l}\text { Número total de empresas da amostra } \\
\text { Para o conjunto de sete indicadores propostos na seção } 2(i=1, \ldots, 7)^{(1)}\end{array}$ \\
\hline
\end{tabular}

Fonte: Elaboração dos autores.

(1) A descrição detalhada pode ser encontrada em IBGE (2008, p. 8).

\subsection{Análise descritiva dos indicadores de resultados e dimensão da inovação}

Os indicadores desta seção examinam a concentração (dispersão), os resultados dos esforços (produto, processo ou organizacional e/ou marketing) e a dimensão da inovação (empresa ou mercado). As informaçōes utilizadas estão sumarizadas nos Gráficos 2 e 3 e completas no Anexo 1.

A taxa de inovação (TI) mede o número de empresas que desenvolveram inovações em produto e/ou processo (em andamento ou abandonados) em relação 
ao total de empresas da IAP. Esse percentual revela diferenças significativas entre os países, como, por exemplo, entre a Alemanha $(63,7 \%$ realizam algum tipo de atividade inovativo) e outros países - Suécia (49\%), Brasil (38\%), França (34\%) e Hungria (17,5\%) - vide Anexo 1. Todavia, um grande número de países tem valores próximos ao médio da amostra (37\%), fato que explica a heterogeneidade não muito elevada do coeficiente de variação $(32,3 \%)$.

\section{GRÁFICO 2}

Taxas de inovação na indústria de alimentos processados

Países selecionados - 2006/2008

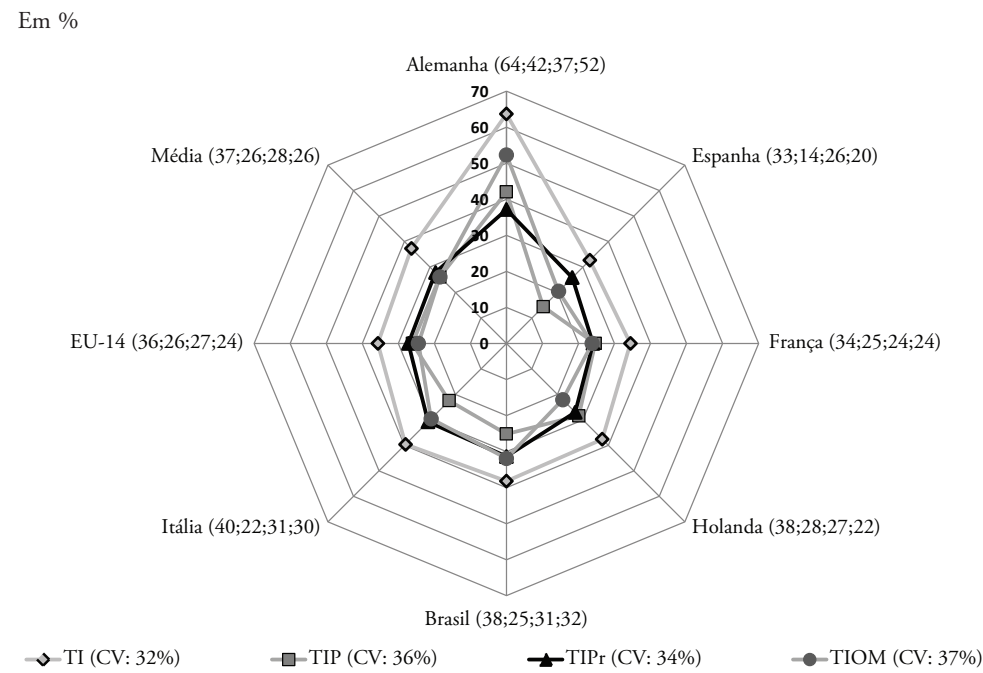

Fonte: CIS (EUROSTAT, 2009) e Pintec (IBGE, 2010). Elaboração dos autores.

Nota: TI: taxa de inovação; TIP: taxa de inovação em produto; TIP: taxa de inovação em processo; TIOM: taxa de inovação organizacional e em estratégias de marketing.

Os números entre parênteses, que seguem a mesma ordem da legenda, representam os valores aproximados de cada indicador para o país.

As inovações (processos, produtos e organizacional e/ou marketing) têm níveis relativamente similares para boa parte da amostra (médias 26\%, 27\% e 26\%, respectivamente), mas estão distribuídas de forma heterogênea devido, em boa medida, aos baixos índices dos países do leste europeu (Anexo 1) e ao elevado desempenho da Alemanha. O Gráfico 2 mostra que a inovação em processo é o tipo mais difundido na indústria (cerca de $28 \%$ da amostra e $75 \%$ das empresas que inovaram). Esses percentuais estão atrelados às características da IAP (escalas produtivas, imitação fácil e adaptações e diferenciação de produtos), que direcionam os esforços das empresas para a ampliação das competências em processo. 
As inovações em produtos, que também exigem das empresas adaptações ou novos métodos produtivos, têm resultados similares aos das inovações em processo (70\% entre as firmas que inovaram). A principal característica dessas inovações, como mostra o Gráfico 3, é o predomínio de "novas para a empresa" - média elevada $(52,5 \%)$ e distribuição homogênea $(24,5 \%)$ - em relação a "novas para o mercado" - média inferior $(38,9 \%)$ e distribuição heterogênea (32\%). Em síntese, parte significativa da inovação na IAP está relacionada com os métodos produtivos para o lançamento de produtos novos no âmbito da empresa (vide seção 3).

Além disso, mesmo que a heterogeneidade não seja muito acentuada (32,4\%) entre os países da amostra para as inovações em produto "novo para o mercado", há casos exemplares de diferenças, como, por exemplo, Holanda $(52,8 \%)$ e Brasil $(10,8 \%)$. Nesse sentido, os resultados (Gráficos 2 e 3) permitem caracterizar o desempenho da IAP do Brasil - pelo reduzido desenvolvimento de produtos novos para o mercado (11\%) - como fortemente "imitativo", ou seja, de absorção de tecnologias na fase de difusão. ${ }^{12}$ Essa é também uma característica da IAP de outros países examinados, mas as assimetrias são menos intensas.

\section{GRÁFICO 3}

Taxas de inovação em produtos para o mercado (IPM) e para a empresa (IPE) na indústria de alimentos processados

Países selecionados - 2006/2008

$\operatorname{Em} \%$

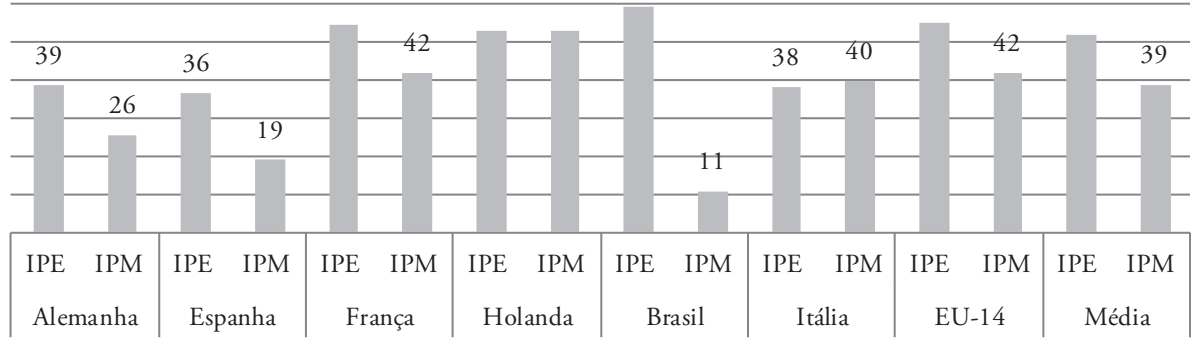

IPE (CV: $25 \%) \operatorname{IPM}(32 \%)$

IPM (CV: 25\%) e IPE: (CV: $32 \%)$

Fonte: CIS (EUROSTAT, 2009) e Pintec (IBGE, 2010). Elaboração dos autores.

Em geral, as duas inovações (produto e processo) demandam mudanças nas estruturas organizacionais e estratégias de marketing, isto é, há certa complementaridade entre elas - razão para a semelhança entre as médias. No Brasil, em particular,

12 Esses resultados são similares aos encontrados por Cabral (1999) e Domingues (2008). 
os três indicadores apresentam valores iguais ou acima da média da amostra, mas inferiores aos de outros países, inclusive de alguns sem uma indústria tão complexa, como, por exemplo, Portugal (as multinacionais têm presença marcante na IAP). Ainda com o intuito de confrontar os resultados e caracterizar os países, a taxa da inovação em estruturas organizacionais e estratégias de marketing da Alemanha é a mais elevada $(52,3 \%)$, o dobro da média da amostra.

\subsection{Indicadores de esforços inovativos: uma análise por agrupamentos}

O objetivo deste item é apresentar o resultado da análise de agrupamentos (clusters) realizada a partir dos indicadores de esforço inovativos (seção 2 e Quadro 1) e aplicada para 18 dos 20 países da amostra - o método adotado exclui os elementos com ausência de informação. Este tipo de análise procura categorizar um conjunto de dados de modo que as observações dentro de um mesmo agrupamento sejam relativamente similares.

Neste estudo, optou-se por uma metodologia em três passos. O primeiro procura formar e determinar o número de grupos por meio do método hierárquico de Ward. Nesse método os agrupamentos são construídos sucessivamente a partir da distância euclidiana entre os elementos (menor distância, maior similaridade), até que seja formado um único grupo que comporte todos os elementos da amostra - ao analista cabe decidir o número de arranjos examinando os testes estatísticos e dendrogramas gerados. Posteriormente, aplicou-se o "método K-médias” (não hierárquico) para (re) classificar de forma mais adequada os elementos de cada agrupamento obtido pelo método anterior - ou seja, procura diretamente a participação de $n$ elementos em $K$ grupos por meio de dois requisitos básicos: semelhança interna e separação dos grupos formados. Em outras palavras, o método hierárquico é utilizado de forma exploratória e os agrupamentos sugeridos são reavaliados pelo método não hierárquico para testar os resultados. ${ }^{13}$ Por fim, esse procedimento foi complementado com a "análise de discriminantes" para obter a função de Fisher que classifica os indicadores segundo sua importância para a formação de cada arranjo.

Dessa forma, a metodologia aplicada permite procurar similaridades entre os países da amostra, tendo como fator de agregação os sete indicadores de esforço para

13 Além de Hair et al. (2005), que defendem este método como o mais apropriado, ver também Gonçalves e Yonamini (2013) e Stallivieri et al. (2010). Este método, que procura minimizar a variância dentro dos grupos formados, tende a resultar em agrupamentos de tamanhos aproximadamente iguais - a cada estágio, juntam-se os dois agrupamentos que representam o menor aumento na soma total dos quadrados. 
inovação, e, posteriormente, avaliar a importância e intensidade desses indicadores na IAP internacional.

O método hierárquico (primeiro passo) possibilita formar 2, 3 ou 4 aglomerados - pela distância descartou-se a formação de um único grupo (vide Dendograma, Anexo 2). O teste K-médias (segundo passo), aplicado sobre estas três possibilidades, garante a seleção de apenas dois arranjos (Anexo 3). ${ }^{14}$ Por fim, para estimar os fatores mais relevantes na formação destes dois agrupamentos, foi realizada a análise de discriminante - vide Tabela 1 (coeficientes mais elevados determinam a importância do indicador no grupo) - para diferenciar os dois grupos selecionados por meio das atividades mais difundidas e, ao mesmo tempo, hierarquizar estas atividades.

\section{TABELA 1}

Função de classificação de Fisher e média dos clusters, segundo atividades inovativas Países selecionados 2006/2008

\begin{tabular}{|c|c|c|c|c|}
\hline \multirow[t]{2}{*}{ Atividades } & \multicolumn{2}{|c|}{$\begin{array}{c}\text { Cluster 1 } \\
\text { (Hungria, Polônia, } \\
\text { Espanha, Brasil, Bulgária, } \\
\text { Holanda, Noruega, França, } \\
\text { Áustria, Croácia, Lituânia, } \\
\text { Eslováquia, Itália e Portugal) }\end{array}$} & \multicolumn{2}{|c|}{$\begin{array}{c}\text { Cluster } 2 \\
\text { (Suécia, Alemanha, Bélgica e } \\
\text { Finlândia) }\end{array}$} \\
\hline & Fisher & Média & Fisher & Média \\
\hline $\begin{array}{l}\text { Aquisição de máquinas, } \\
\text { equipamentos e softwares }\end{array}$ & 0,36 & 21,03 & 0,54 & 39,02 \\
\hline$P \& D$ & 0,08 & 12,45 & 0,33 & 32,15 \\
\hline Treinamento & $-0,18$ & 13,64 & $-0,42$ & 24,4 \\
\hline $\begin{array}{l}\text { Introdução de inovações } \\
\text { tecnológicas no mercado }\end{array}$ & 0,18 & 10,53 & 0,16 & 21,45 \\
\hline $\begin{array}{l}\text { Projetos industriais e outras } \\
\text { preparaçôes técnicas }\end{array}$ & 0,51 & 9,33 & 1,04 & 16,54 \\
\hline Aquisição de P\&D externa & 0,07 & 5,29 & $-0,13$ & 15,67 \\
\hline $\begin{array}{l}\text { Aquisição de outros } \\
\text { conhecimentos externos }\end{array}$ & $-0,08$ & 4,51 & 0,82 & 21,56 \\
\hline (Constant) & & $-6,66$ & & $-30,14$ \\
\hline
\end{tabular}

Fonte: CIS (EROSTAT, 2009) e Pintec (IBGE, 2010). Elaboração dos autores com base no software SPSS.

As atividades projeto industrial e outras preparações técnicas e aquisição de máquinas, equipamentos e softwares são as que mais contribuem para a formação

14 Para verificar o número de grupos mais apropriado pelo método de K-médias, foram comparadas as (mais elevadas) "distâncias" F para a maioria dos indicadores nos diferentes agrupamentos (HAIR et al., 2005). Em todas as simulaçōes adotou-se o valor de $5 \%$ de significância. 
de ambos os aglomerados (vide Fisher na Tabela 1 - grupo 1: 0,51 e 0,36; grupo 2: 1,04 e 0,54, respectivamente) - no arranjo 2 há também a aquisição de outros conhecimentos externos. No entanto, se a segunda dessas atividades é a mais difundida em ambos os grupos (médias elevadas de 21,03 e 39,02, respectivamente), a primeira é apenas a sexta mais praticada pelos países do grupo 2. Assim, a aquisição de máquinas, equipamentos e softwares parece ser o fator determinante para a formação dos dois ajuntamentos - resultado compatível com os alcançados com as inovações em processo da seção anterior.

Os resultados da Tabela 1 permitem duas observações adicionais. A primeira refere-se às assimetrias entre as médias dos dois blocos de países: o aglomerado 1 apresenta valores significativamente inferiores aos do grupo 2, ou seja, as empresas da IAP deste último grupo praticam maior número de atividades tecnológicas e de forma mais disseminada. A segunda observação, que reforça a argumentação da seção 2, é que a P\&D "tradicional” não figura como a atividade mais relevantes para a união de cada um dos dois aglomerados ( $4^{a}$ nos dois grupos), nem a mais difundida ( $3^{\mathrm{a}}$ e $2^{\mathrm{a}}$, respectivamente). Este resultado permite confirmar a hipótese de que as atividades inovativas consideradas neste estudo são, de fato, relevantes para a indústria.

Em razão do grande número de países no primeiro arranjo e da discrepância entre as médias dos indicadores nos dois agrupamentos, a metodologia anterior foi aplicada ao primeiro grupo para evidenciar assimetrias. A distribuição $\mathrm{F}$, decorrente do emprego do método da K-média, indica a segmentação do grupo 1 em quatro outros (ver Anexo 4). A Tabela 2 mostra os resultados da função de Fisher para as atividades em cada um dos subgrupos.

Os resultados da Tabela 2 mantêm a aquisição de máquinas, equipamentos e softwares como atividade mais relevante para determinação dos quatro subgrupos, seguida pela P\&D interna. No entanto, estas atividades incidem de forma muito distinta em cada arranjo: cluster 1.1 - as duas médias são baixas; cluster 1.2 - apesar da semelhança entre as duas médias, P\&D é mais praticada ${ }^{15}$ e emerge a atividade projeto industrial e outras preparações técnicas como determinante (identicamente ao aglomerado 2, Tabela 1); clusters 1.3 e 1.4 (Brasil é inserido) - a determinação desses dois grupos está fortemente atrelada à aquisição de máquinas, equipamentos e softwares e secundariamente à $P \& D$.

15 A Holanda (Unilever) e a França (Danone) possuem empresas líderes mundiais na IAP, fato que ajuda a explicar a força desses países em atividades de P\&D interna $-2^{\mathrm{a}}$ e $5^{\mathrm{a}}$ maiores empresas de alimentos do mundo, respectivamente (FORBES, 2011). 
TABELA 2

Função de classificação de Fisher e média dos clusters para subgrupos do grupo 1 , segundo atividades inovativas

Países selecionados - 2006/2008

\begin{tabular}{|c|c|c|c|c|c|c|c|c|}
\hline \multirow[t]{2}{*}{ Atividades } & \multicolumn{2}{|c|}{$\begin{array}{c}\text { Cluster } 1.1 \\
\text { Espanha, } \\
\text { Bulgária, Hungria, } \\
\text { Polônia }\end{array}$} & \multicolumn{2}{|c|}{$\begin{array}{c}\text { Cluster } 1.2 \\
\text { França, Holanda, } \\
\text { Lituânia, Noruega }\end{array}$} & \multicolumn{2}{|c|}{$\begin{array}{c}\text { Cluster } 1.3 \\
\text { Itália e Portugal }\end{array}$} & \multicolumn{2}{|c|}{$\begin{array}{c}\text { Cluster } 1.4 \\
\text { Brasil, Áustria, } \\
\text { Eslováquia e } \\
\text { Croácia }\end{array}$} \\
\hline & Fisher & Média & Fisher & Média & Fisher & Média & Fisher & Média \\
\hline $\begin{array}{l}\text { Aquisição de } \\
\text { máquinas, } \\
\text { equipamentos e } \\
\text { softwares }\end{array}$ & 4,05 & 13,37 & 3,05 & 18,64 & 16,69 & 35,44 & 4,92 & 23,87 \\
\hline$P \& D$ & 1,50 & 5,83 & 1,99 & 19,70 & 6,36 & 13,98 & 1,75 & 11,04 \\
\hline Treinamento & $-0,31$ & 6,10 & $-0,44$ & 15,88 & $-0,23$ & 21,22 & $-0,38$ & 15,13 \\
\hline $\begin{array}{l}\text { Introdução de } \\
\text { inovaçôes tecnológicas } \\
\text { no mercado }\end{array}$ & $-1,56$ & 5,86 & $-0,25$ & 11,83 & $-9,81$ & 8,54 & $-0,66$ & 14,90 \\
\hline $\begin{array}{l}\text { Proj. industriais e } \\
\text { outras preparaçốes } \\
\text { técnicas }\end{array}$ & $-0,44$ & 5,05 & 3,05 & 11,84 & $-5,74$ & 10,28 & 1,02 & 10,64 \\
\hline $\begin{array}{l}\text { Aquisição de P\&D } \\
\text { externa }\end{array}$ & $-1,63$ & 3,28 & $-1,12$ & 8,26 & $-8,27$ & 4,23 & $-1,45$ & 4,85 \\
\hline $\begin{array}{l}\text { Aquisição de outros } \\
\text { conhecimentos } \\
\text { externos }\end{array}$ & $-0,28$ & 2,78 & 0,44 & 5,73 & $-4,79$ & 2,95 & 0,32 & 5,80 \\
\hline (Constant) & & $-23,01$ & & $-59,00$ & & $-243,29$ & & $-64,67$ \\
\hline
\end{tabular}

Fonte: CIS (EROSTAT, 2009) e Pintec (IBGE, 2010). Elaboraçăo dos autores com base no software SPSS.

Em síntese, a aquisição de máquinas, equipamentos e softwares é o principal determinante na formação dos aglomerados. Nos arranjos 2 e 1.2, projeto industrial e outras preparaçôes técnicas e, especialmente, a P\&D apresentam-se também como atividades relevantes. Nos outros grupos, a P\&D é praticada em níveis relativamente mais baixos. Ademais, nos grupos de países com maiores taxas de P\&D, outras atividades são mais densamente praticadas, aparentando certa complementaridade entre os esforços tecnológicos e ambiente.

Por outro lado, a dispersão assimétrica do conjunto de atividades inovativas da IAP permite hierarquizar os arranjos de países alcançados (ordem decrescente): 2, $1.2,1.3,1.4$ e 1.1. Aceitos tais resultados, além de guardar relação com a dispersão das atividades inovativas, essa classificação parece seguir o nível de desenvolvimento econômico e tecnológico dos países. 


\section{Conclusões}

Em boa medida as atividades inovativas na IAP estão pautadas nas mudanças nos métodos e escalas produtivas e na complementaridade dos processos de adaptação e difusão tecnológica. Esta relação exige das empresas tanto interações com indústrias diferentes, quanto distintos modos de aprendizado e acúmulo de conhecimento que auxiliam no desenvolvimento das capacidades técnicas da firma. Os elementos de busca (NELSON; WINTER, 1982, cap. 11) estão por um lado, relacionados com a assimilação e adaptação de tecnologias desenvolvidas por outros agentes externos que são incorporadas e aperfeiçoadas pela firma. Por outro lado, as decisões que norteiam o esforço de P\&D interna estão associadas com a incorporação de tecnologias e a diferenciação de produtos, ou seja, com as inovações incrementais e "arquitetônicas". Nessas circunstâncias, o desenvolvimento sistêmico de produtos e/ou processos produtivos aperfeiçoados ou mais sofisticados é um processo complexo que requer conhecimentos "prático", técnico e codificado e capacitaçôes para combinar eficientemente conhecimentos internos e externos, aspectos que também caracterizam outras indústrias similares.

Apesar de limitado a poucas empresas, o estudo sobre os depósitos de patentes confirma que algumas das empresas analisadas desenvolvem (e codificam) conhecimento em áreas fora da sua base de conhecimento. Essas pesquisas permitem aumentar as possibilidades de inovaçôes incrementais e de diversificaçōes em áreas de negócios próximas, bem como auxiliam e fomentam os desenvolvimentos de inovações geradas pela relação usuário-produtor e produtor-cliente. Por outro lado, essas atividades não só produzem, mas também demandam conhecimentos em vários campos científicos. Considerando que mesmo para as empresas líderes mundiais é inviável a permanente capacitação em muitas áreas científicas, os esforços tecnológicos acabam concentrados em determinados campos - transportes, conservação de produtos, etc.

Os indicadores de inovação e atividades inovativas contribuíram para a discussão de forma distinta. Eles foram analisados a partir de três aspectos. Os dois primeiros (resultados e dimensões das inovaçôes) procuraram mostrar o foco e a abrangência das inovações na IAP. Nesse caso, os indicadores do presente estudo apontam que o principal tipo de inovação é "em processo" e "em produto novo para a empresa".

Os indicadores obtidos mostram heterogeneidade entre os países, fato que pode estar associado a questôes técnicas, estruturais da IAP - empresas de diferentes 
tamanhos, segmentos com poucas barreiras à entrada, etc. - e institucionais (sistemas nacionais de inovação). Por isso, as diferenças permitem avaliar as características da IAP nos países da amostra. Por exemplo, a IAP brasileira, que apresenta taxas de inovação em processo acima da média dos países analisados e taxa de inovação em produto inferior, pode ser classificada como voltada para inovaçóes "em processo" ou "redutoras de custos". Esses resultados permitem sugerir que a IAP do Brasil tem um comportamento "imitativo", absorvendo tecnologias na fase de difusão. Essa avaliação, com alguns elementos teóricos adicionais (tarefa não realizada neste estudo), pode caminhar para uma metodologia sobre determinação do papel (ou padrôes) da indústria de cada país no contexto internacional.

A atenção dada aos esforços para inovação foi o terceiro aspecto da contribuição dos indicadores deste estudo. O perfil esboçado acima é consistente com a análise de clusters. O Brasil, por exemplo, está entre os países que menos pratica P\&D, fato que colabora para a baixa taxa de inovação "para o mercado" e percentual elevado na atividade aquisição de máquinas, equipamentos e software. Ressalte-se que esta última é o principal destaque entre as atividades inovativas da amostra.

A P\&D é uma atividade pouco praticada na maioria dos países da amostra, mas, nos países em que ela é mais intensa, outras atividades - especialmente projeto industrial e outras preparações técnicas para a produção e distribuição - também são realizadas. Esse resultado parece descrever um gradiente entre aprendizado, conhecimentos e capacitações.

Em suma, os resultados confirmam a relevância de uma avaliação a partir das atividades inovativas e permitem estabelecer considerações sobre a IAP em três aspectos: um exame dos esforços e dos resultados para a inovação na indústria; tratar de aspectos conexos e complementares que auxiliam no desempenho inovativo da IAP; e comparar o desempenho tecnológico de diferentes países.

\section{Referências bibliográficas}

ACHA, V.; VON TUNZELMANN, N.; Innovation in "low-tech" industries. In: FAGERBERG, J.; MOWERY, D.; NELSON, R. R. (Eds). The Oxford handbook of innovation. Oxford: Oxford University Presst, 2005, p. 407-432.

ALFRANCA, O.; RAMA, R.; VON TUNZELMANN, N. Innovation in food and beverage multinationals. In: RAMA, R. (Ed.) Multinational agribusiness. NY, London, Oxford: Food Products Press an imprint of The Haworth Press, Inc., 2005. 
CABRAL, J. E. O. Patterns and determinants of technological innovation in the Brazilian food industry. Doctor Thesis - Department of Agricultural and food Economics. Londres: University of Reading, 1999.

CHRISTENSEN, J. F. Asset profiles for technological innovation. Research Policy, v. 24, n. 5, p. 727-745, 1995 .

DOMINGUES, S. A. A indústria de alimentos e bebidas no Brasil: uma análise da dinâmica tecnológica e das estratégias de inovação de suas empresas entre 1998 e 2005. Tese (Doutorado). Campinas: Unicamp, 2008.

EUROSTAT. Community Innovation Survey - CIS. 2009. Disponível em: <http://epp.eurostat. ec.europa.eu/portal/page/portal/eurostat/home> (versão revisada). Acesso em: 17 jul. 2012.

FILIPPAIOS, F.; RAMA, R. cultural distance and internationalization: the world's largest food and drink multinationals. Agribusiness, v. 27, n. 4, p. 399-419, 2011.

FORBES. Global 2000 leading companies. 2011. Disponível em: <http://www.forbes.com/ lists/2012/18/global2000_2011.html>. Acesso em: 17 jul. 2012.

FURTADO, A. T.; CARVALHO, R. de Q. Padrōes de intensidade tecnológica da indústria brasileira: um estudo comparativo com os países centrais. São Paulo em Perspectiva, v. 19, n. 1, p. 70-84, mar. 2005.

FURTADO, A. T. Difusão tecnológica: um debate superado? In: PELAEZ, V.; SZMRECSÁNYI, T. (Orgs.). Economia da inovação. São Paulo: Editora Hucitec/Ordem dos Economistas do Brasil, 2006, p. 168-192.

GOMES, R. Empresas transnacionais e internacionalização da P\&D. São Paulo: Editora da Unesp, 2006.

GONÇALVES F. O.; YONAMINI, F. M. Em busca de uma nova taxonomia de regimes tecnológicos para a indústria de transformação brasileira. Revista EconomiA, v. 14, n. 1A, p. 145-158, jan./abr. 2013.

HAIR, J. F.; TATHAM, R. L.; ANDERSON, R. E.; BLACK, W. C. Análise multivariada de dados. Tradução de Adonai Schlup Sant'Ana e Anselmo Chaves Neto. 5 ed. Porto Alegre: Bookman, 2005.

HIRSCH-KREINSEN, H. "Low-Technology": a forgotten sector in innovation policy. J. Technol. Manag. Innov., v. 3, n. 3, p 11-20, 2008.

HENDERSON, R. M.; CLARK, K. B. Architectural innovation: the reconfiguration of existing product technologies and the failure of established firms. Administrative Science Quarterly, v. 35, p. 9-30, 1990. 
IBGE - Instituto Brasileiro de Geografia e Estatística. Pesquisa de Inovação Tecnológica Pintec. Rio de Janeiro: IBGE, 2010. Disponível em:<http://www.pintec.ibge.gov.br/> Acesso em: 17 jul. 2012.

Notas Técnicas da Pesquisa de Inovação Tecnológica - Pintec. Rio de Janeiro: IBGE, 2008. Disponível em: <http://www.pintec.ibge.gov.br/> Acesso em: 17 jul. 2012.

KLINE, S. J.; ROSENBERG, N. An overview of innovation. In: LANDAU, R.; ROSENBERG, N. (Eds.). The positive sum strategy - harnessing technology for economic growth. Washington, 1986, p. 275-305.

ISI. Web of Knowledge - Derwent Innovations Index, 2013. Disponível em: <http://portal. isiknowledge.com/>.

MENDONÇA, S. Brave old world: Accounting for "high-tech" knowledge in "low-tech" industries. Research Policy, n. 38, p. 470-482, 2009.

NELSON, R.; WINTER, S. An evolutionary theory of economic change. Cambridge, MA: Harvard University Press, 1982.

OCDE. ISIC REV. 3 Technology Intensity Definitionanberd and STAN. Julho 2011. Disponível em: <http://www.oecd.org/dataoecd/43/41/48350231.pdf> Acesso em: 17 jul. 2012.

OECD. Manual de Oslo: diretrizes para coleta e interpretação de dados sobre inovação. OECD, 2005.

PATEL, P.; PAVITT, K. The technological competencies of the world's largest firms: complex and path-dependent, but not much variety. Research Policy, n. 26, p. 141-156, 1997.

PAVITT, K. Sectoral patterns of technical change: towards a taxonomy and a theory. Research Policy, n. 13, p.343-373, 1984

PENROSE, E. The theory of the growth of the firm. $3^{\text {rd }}$ ed. Oxford, 1959.

RAMA, R. (Org) Handbook of innovation in the food and drink industry. New York: Howarth Press, 2008.

RAUD, C. Os alimentos funcionais - a nova fronteira da indústria alimentar: análise das estratégias da Danone e da Nestlé no mercado brasileiro de iogurtes. Revista Sociologia e Política, v. 16, n. 31, p. 85-100, 2008.

ROBERTSON, P. L.; SMITH, K. H.; VON TUNZELMANN, N. Innovation in low- and medium-technology industries. Research Policy, v. 38, n. 3, p. 441-446, 2009.

ROSENBERG, N. Por dentro da caixa preta: tecnologia e economia. Campinas: Editora da Unicamp, 1982. 
SMITH, K. What is the "knowledge economy"? Knowledge-intensive industries and distributed knowledge bases. In: Druid Summer Conference on the Learning Economy - Firms, Regions and Nation Specific Institutions. Aalborg, Dinamarca, junho 2000.

STALLIVIERI F.; BRITTO, J.; CAMPOS, R.; VARGAS, M. Padrôes de aprendizagem, cooperação e inovação em aglomerações produtivas no Brasil: uma análise multivariada exploratória. Economia, v. 11, n. 1, p. 125-154, 2010.

VON HIPPEL, E. Democratizing innovation. Cambridge, MA: MIT Press, 2005. 


\section{Anexos}

Anexo 1 - Valores dos indicadores TI, TIP, TIPr, TIOM, IPE e IPM

\begin{tabular}{|c|c|c|c|c|c|c|}
\hline Países & TI & TIP & TIPr & TIOM & IPE & IPM \\
\hline Alemanha & 63,7 & 42,1 & 37,2 & 52,3 & 38,7 & 25,5 \\
\hline Espanha & 32,7 & 14,4 & 25,8 & 20,5 & 36,5 & 18,9 \\
\hline França & 34,4 & 24,6 & 24,0 & 23,8 & 54,8 & 42,0 \\
\hline Holanda & 37,6 & 28,4 & 27,0 & 22,1 & 52,8 & 53,0 \\
\hline Brasil & 38,2 & 25,1 & 31,4 & 31,9 & 59,2 & 10,8 \\
\hline Itália & 39,6 & 22,5 & 30,8 & 29,6 & 38,1 & 39,9 \\
\hline EU-14 & 35,7 & 26,1 & 27,2 & 24,4 & 54,9 & 41,9 \\
\hline Bélgica & 48,6 & 33,3 & 39,2 & 36,2 & 43,2 & 44,4 \\
\hline Bulgária & 29,6 & 20,1 & 19,4 & 17,9 & 46,7 & 28,5 \\
\hline Lituânia & 32,0 & 26,7 & 27,5 & 24,8 & 58,3 & 46,7 \\
\hline Hungria & 17,5 & 14,2 & 9,3 & 12,2 & 74,5 & 42,0 \\
\hline Malta & 40,4 & 27,7 & 36,2 & 27,7 & 26,3 & 42,1 \\
\hline Áustria & 34,6 & 25,1 & 24,6 & 23,3 & 57,5 & 47,7 \\
\hline Polônia & 17,8 & 13,1 & 12,9 & 10,1 & 49,9 & 38,4 \\
\hline Portugal & 49,5 & 32,1 & 43,7 & 35,6 & 49,5 & 32,5 \\
\hline Romênia & 20,0 & 12,7 & 17,6 & 15,4 & 55,4 & 21,1 \\
\hline Eslováquia & 34,7 & 28,1 & 24,8 & 23,6 & 56,2 & 59,9 \\
\hline Finlândia & 55,8 & 49,1 & 45,7 & 37,4 & 79,7 & 54,4 \\
\hline Suécia & 49,1 & 35,0 & 31,9 & 28,1 & 55,6 & 45,7 \\
\hline Noruega & 41,1 & 28,4 & 21,9 & 27,5 & 69,1 & 37,8 \\
\hline Croácia & 28,6 & 19,9 & 26,0 & 21,9 & 47,0 & 45,9 \\
\hline Média & 37,3 & 26,1 & 27,8 & 26,1 & 52,5 & 38,9 \\
\hline $\mathrm{Cv}$ & 32,3 & 36,1 & 34,1 & 37,1 & 24,5 & 32,4 \\
\hline
\end{tabular}

Fonte: CIS (EUROSTAT, 2009) e Pintec (IBGE, 2010). Elaboração dos autores. 
Anexo 2 - Agrupamento de países: similaridade por esforço inovativo

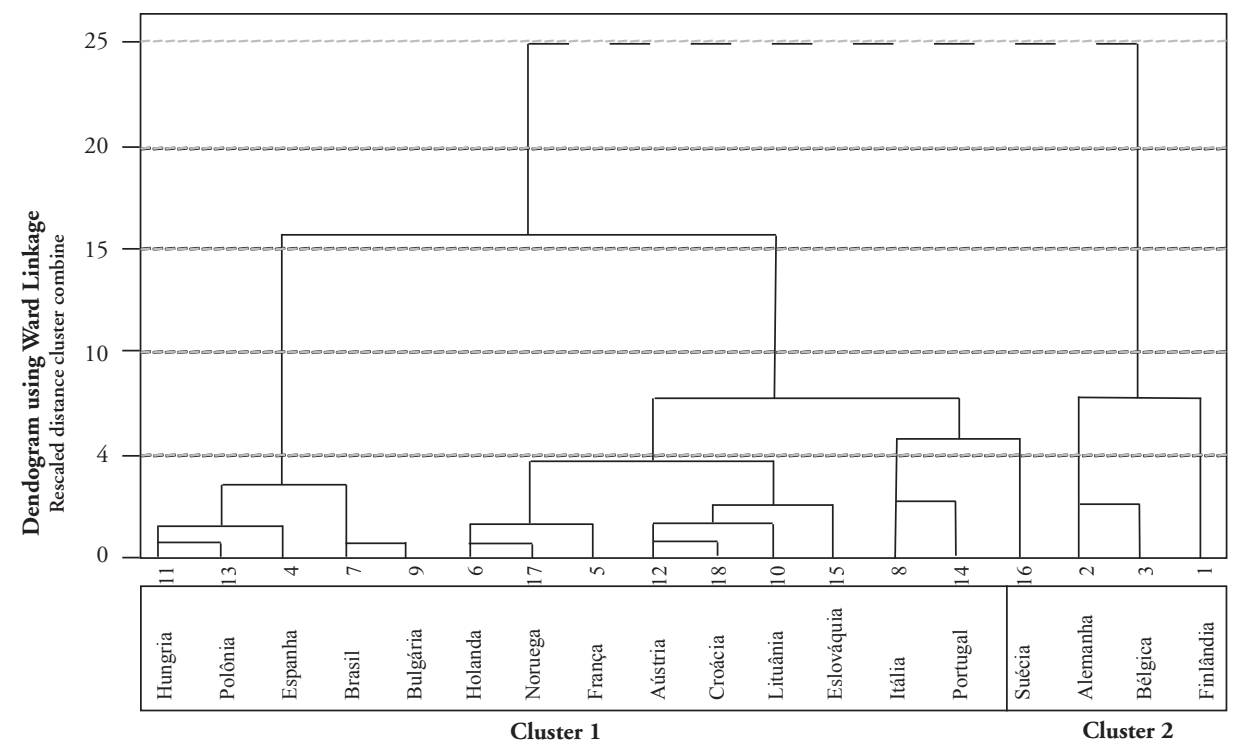

Fonte: CIS (EROSTAT, 2009) e Pintec (IBGE, 2010). Elaboração dos autores com base no software SPSS.

Anexo 3 - Distância F e significância dos fatores na formação dos clusters

\begin{tabular}{|c|c|c|c|c|c|c|}
\hline \multirow[b]{2}{*}{ Atividades } & \multicolumn{2}{|c|}{2 Clusters } & \multicolumn{2}{|c|}{3 Clusters } & \multicolumn{2}{|c|}{4 Clusters } \\
\hline & $\mathbf{F}$ & Sig. & F & Sig. & F & Sig. \\
\hline $\begin{array}{l}\text { Aquisição de máquinas, } \\
\text { equipamentos e softwares }\end{array}$ & 18,15 & 0,00 & 12,11 & 0,00 & 8,89 & 0,00 \\
\hline$P \& D$ & 21,63 & 0,00 & 11,03 & 0,00 & 9,27 & 0,00 \\
\hline Treinamento & 6,36 & 0,02 & 10,52 & 0,00 & 19,82 & 0,00 \\
\hline $\begin{array}{l}\text { Introdução de inovações } \\
\text { tecnológicas no mercado }\end{array}$ & 17,90 & 0,00 & 7,59 & 0,01 & 12,37 & 0,00 \\
\hline $\begin{array}{l}\text { Projetos industriais e outras } \\
\text { preparaçōes técnicas }\end{array}$ & 7,24 & 0,02 & 2,83 & 0,09 & 13,43 & 0,00 \\
\hline Aquisição de P\&D externa & 14,18 & 0,00 & 8,86 & 0,00 & 8,56 & 0,00 \\
\hline $\begin{array}{l}\text { Aquisição de outros } \\
\text { conhecimentos externos }\end{array}$ & 53,54 & 0,00 & 14,38 & 0,00 & 23,59 & 0,00 \\
\hline
\end{tabular}

Fonte: CIS (EROSTAT, 2009) e Pintec (IBGE, 2010). Elaboração dos autores com base no software SPSS. 
Anexo 4 - Distância F e significância dos fatores no reagrupamento do cluster 1

\begin{tabular}{|c|c|c|c|c|c|c|}
\hline \multirow{2}{*}{ Atividades } & \multicolumn{2}{|c|}{2 Clusters } & \multicolumn{2}{|c|}{3 Clusters } & \multicolumn{2}{|c|}{4 Clusters } \\
\hline & F & Sig. & $\mathbf{F}$ & Sig. & $\mathbf{F}$ & Sig. \\
\hline $\begin{array}{l}\text { Aquisição de máquinas, } \\
\text { equipamentos e softwares }\end{array}$ & 7,44 & 0,02 & 36,30 & 0,00 & 42,85 & 0,00 \\
\hline $\mathrm{P} \& \mathrm{D}$ & 3,14 & 0,10 & 1,33 & 0,31 & 6,27 & 0,01 \\
\hline Treinamento & 27,82 & 0,00 & 10,38 & 0,00 & 4,52 & 0,03 \\
\hline $\begin{array}{l}\text { Introdução de inovaçôes } \\
\text { tecnológicas no mercado }\end{array}$ & 4,60 & 0,05 & 13,05 & 0,00 & 6,97 & 0,01 \\
\hline $\begin{array}{l}\text { Projetos industriais e outras } \\
\text { preparaçóes técnicas }\end{array}$ & 6,19 & 0,03 & 5,48 & 0,02 & 12,83 & 0,00 \\
\hline Aquisição de P\&D externa & 0,03 & 0,87 & 0,09 & 0,91 & 1,39 & 0,30 \\
\hline $\begin{array}{l}\text { Aquisição de outros } \\
\text { conhecimentos externos }\end{array}$ & 1,86 & 0,20 & 4,15 & 0,05 & 1,85 & 0,20 \\
\hline
\end{tabular}

Fonte: CIS (EROSTAT, 2009) e Pintec (IBGE, 2010). Elaboração dos autores com base no software SPSS. 
